\section{Some Things Are Better Left Unsaid}

\author{
M. Castillo, Editor-in-Chief
}

My ideas seem to frighten you. . . some things are better left unsaid. Daryl Hall and John Oates

l: law enforcement, as in radiology, it is customary to ask a witness (in our case our trainees) to repeatedly describe an observed event (in our situation the imaging findings). Sometimes we ask our trainees to describe the features of different lesions even when they are not looking at images. This is based on our belief that repeated verbal processing builds mental images that we can all later retrieve in an attempt to match them with what we are seeing on our studies. We all have been taught this way, and because of this, we think it is a good method and continue to use it, but, are we doing the right thing?

In 1990, two psychologists from the Universities of Pittsburgh and Washington noticed that previous verbal articulation distorted future visual recognition, a phenomenon they named "verbal overshadowing." ${ }^{1}$ For their dissertations, they tested several hypotheses, one being that verbalizing the appearance of something previously seen impaired its future recognition (this is exactly what I mistakenly ask my residents to do: "Describe for me the imaging findings of the glioblastoma we saw last week"). Schooler* and Engstler-Schooler did a series of simple tests, and one went like this: The participants were shown a face; some were asked to repeatedly describe that face and a control group was not asked anything. Later the same face was shown to all participants_-guess who did better? The rehearsed group did much worse! It seems that the brain has specific systems that help it remember faces, a common task needed for socialization. A well-known condition that impairs this task is prosopagnosia, in which subjects have varying inabilities to recognize faces. More interesting, our brain recognizes faces more easily when they are in the usual orientation. If faces are presented upside down, we have trouble recognizing them. Repeated verbalization also decreases the chances of correctly recognizing a face. Further experiments determined that when visual stimuli are difficult to verbalize, the memory of them is actually impaired instead of improved by repeated verbalization. Perception of color is also affected by verbal descriptions. When shown colors and then asked to describe them, subjects ended up choosing the one that best fit their description rather than the one they were initially shown.

This makes me wonder if persistently asking our trainees to describe the signal intensity of lesions may actually be detrimental to future information retrieval and interpretation of images. What about our trainees reporting the same findings over and over again by dictating them? Does this improve their (our) memory? What we radiologists do is to verbalize something that is nonverbal: our perception of images. It is known that verbalizing stimuli facilitates memory but only if the stimuli match our ability to use language to describe them; in this way, both effects are

\footnotetext{
* Dr. Jonathan Schooler is now Professor of Psychology at the University of California in Santa Barbara. His original article (1990) on verbal overshadowing has been quoted more than 400 times in the scientific literature.

http://dx.doi.org/10.3174/ajnr.A3319
}

concordant and additive, and memory is facilitated. Thus, rehearsing something like a written history lesson may help us do better on a written examination because in this situation there are no images, only words. In a different experiment, subjects were given a simple verbal statement, and some were asked to repeat it out loud while others were not. In this situation, memory retrieval was improved in those who verbalized the statement, but curiously the improvement was only marginal. Could it be that dictating the same findings innumerable times during our lifetimes as radiologists only minimally helps us to be better professionals?

Not only does verbalizing interfere with immediate memory recall but it affects our long-term memory. There is, however, hope for radiologists: By limiting the time we need to recognize an image, the effects of verbal overshadowing decrease. Therefore, it may be better to interpret cases quickly, rather than taking too much time to think about them! As our schedules become more complicated and full, we spend less time interpreting images, and it is possible that this paradoxically increases our accuracy rather than decreasing it as commonly thought. It is also true that waiting longer between verbalization and re-evaluation of images reduces the effects of overshadowing. ${ }^{2}$ Both Schooler and Engstler-Schooler went on to publish several more articles that confirmed their initial observations, which they named the "recoding interference hypothesis." This hypothesis basically states that verbalizing a visual memory produces a biased memory representation that interferes with the original visual memory and its future recognition. To me, the implications of these observations for radiologists are staggering and deserve a closer look.

In 2001, two different investigators published a meta-analysis of 29 verbal overshadowing experiments performed in 2000 participants. ${ }^{3}$ This analysis included only studies dealing with face recognition, and the authors concluded that the data clearly indicated that subjects who described a face were much more likely to misidentify it subsequently compared with those who did not generate a description before identification. It appears that we self-generate misinformation and manipulate its output, leading the subsequent recognition errors. ${ }^{4}$ To complicate things even further, if participants in a study are given elaborate and detailed instructions as to what to recall, they show poorer results than those who are given the right to free recall. Again, as it pertains to radiology, are we harming ourselves by demanding from our trainees highly structured and detailed reports for each study they interpret? Would a more accurate representation of imaging findings occur in free-form reports?

Evidence exists that verbal overshadowing extends beyond face and color recognition to also affect wine tasting, decision-making, voice recognition, and insight problem-solving. ${ }^{4}$ An extension of the face recognition studies looked at the influence of verbal overshadowing with respect to attractiveness. ${ }^{5}$ These investigators found that individual perception of attractiveness was highly influenced by the amount of verbal attention it received. This influence was most prominently found in females compared with males. It seems that women can change their perception of human face attractiveness by verbalizing. In my experience, this makes sense because we men are more rigid in our perception of beauty and rarely change our initial impressions of it. Verbal overshadowing extends to taste, and as such, 
food (and wine) generally does not taste exactly the same way it did the first time we tried it. I know that after tasting something I really liked and talking about it for weeks, the second taste is always surprisingly different and many times disappointing, though this could be due to differences in preparations.

Because visual memory representations incorporate visual, spatial, and temporal characteristics, studying a set of images (such as CT/MR images consecutively displayed in sets of 6,9 , or 12 images on each monitor) is very different from studying individual images presented in sequence (such as "stacking" CT/MR images in the monitor). Learning sequences of images recruits the verbal system (and thus verbal overshadowing); something that does not occur with individual pictures. So, it is possible that looking at images the way they were traditionally displayed on film (sequentially) is counterproductive. I consider myself a good clinical neuroradiologist but hate looking at images that are stacked. Regardless of that, my interpretations are generally correct, consistent, and fast. After thinking about it, I realize that I do not look at images sequentially but look at the 9 images per monitor at the same time as if they were a single picture. This allows me to be very fast, and this speed in combination with the nonsequential evaluation may decrease my verbal overshadowing and permit me to recognize abnormalities faster and easier. Additionally, I never try to convince myself that I know what the studies show; and because trainees dictate all of my reports, the effects of verbal overshadowing are minimized. Investigations have shown that if one separates the visual from the spatial from the temporal characteristics in an image, the effects of verbal overshadowing are prevented or attenuated. ${ }^{6}$ It seems that our brains retain images and verbal representations at different rates, and both are available to use differently also. Because most research has been done by using static images (pictures), some argue that these do not adequately replicate reality that is characterized by fluidity (thus using videos may be a better choice). ${ }^{7}$

Environmental stimuli are of 2 types: static and dynamic. I have already discussed the effect of verbal overshadowing on static images, so I will now concentrate on events that happen in a dynamic fashion. Events consist of objects in situations characterized by a constant change in spatial relations, mainly distances and orientations, with time. ${ }^{8}$ While verbalization of static images may be considered as concrete, verbalization of events is abstract. If we put an event into words, we end up with 2 competing models: a verbal description and an observation. These 2 models interfere with each other, and that is why events never occurred the way we describe them initially. Not only are our initial verbal descriptions different from the event itself, but subsequent verbal descriptions are different from the initial ones. People who were asked to write down what they saw on September 11, 2001, were asked again several years later to rewrite their impressions, and the 2 versions were so different that many complained that what was given to them as their initial version was false. The reverse is true: You see what you want to see. Individuals asked to verbally describe an unseen event will shape their perception of it by recognizing mostly the parts previously described (these parts are said to become "prioritized"). This may not be all that bad. Verbalbefore-event descriptions help us to identify distractors, allowing us to concentrate on what is important. This observation applies only to dynamic events and does not improve recognition of static images. Thus, teaching our trainees what is important to look for in static images is probably not beneficial; however, teaching them what to look for in dynamic studies such as cerebral angiograms is very useful because it filters out the "noise."

How can we attenuate the effects of verbal overshadowing? All of us have seen (on television, at the movies) hypnosis used by law enforcement agencies (and spies) to increase recall. If one can eliminate the tainted consciousness, perhaps tapping the unconscious will result in better recollections. Studies have looked at this issue and show that the opposite is true; that is, hypnosis actually decreases accuracy, leads to false confidence, and increases suggestibility leading to the procurement of misleading information. ${ }^{9}$ Solutions to verbal overshadowing may be simpler: Avoid too much verbalization, after verbalization engage in a nonverbal task, increase the length of time between verbalization and image recognition, try to verbalize your impression of dynamic events, use free-form verbal expressions rather than highly structured ones, and avoid describing colors. ${ }^{10}$ Another observation that I find of tremendous importance to us is the effect of overshadowing on the type of recall required. It seems that if you ask individuals for "piecemeal" descriptions and recollections, overshadowing of subsequent recognition is much greater than if you ask them for "elaborative" descriptions. This brings me to the last part of this essay: Most books from which we study nowadays present information in a bulleted or piecemeal fashion rather than a long prose form. Because subsequent image discrimination is more affected by previous elaboration of piecemeal descriptions, are we still learning the right way? Would it be better to go back and read books that present information in an elaborative fashion?

Okay, maybe I am exaggerating, but I am trying to play the devil's advocate by bringing up these issues regarding verbal overshadowing. It just seems that they are important enough that perhaps further investigation into how they apply to radiology may be needed. Next month's Perspectives will deal with thoughts about the use and misuse of the scientific process.

\section{REFERENCES}

1. Schooler JW, Engstler-Schooler TY. Verbal overshadowing of visual memories: some things are better left unsaid. Cogn Psychol 1990;22:36-71

2. Finger K, Pezdek K. The effect of the cognitive interview on face identification accuracy: release from verbal overshadowing. J Appl Psych 1999;84:340-48

3. Meissner CA, Brigham JC. A meta-analysis of the verbal overshadowing effect in face identification. Applied Cog Psych 2001;15:603-16

4. Meissner CA, Memon A. Verbal overshadowing: a special issue exploring theoretical and applied issues. Applied Cog Psych 2002;16:869-72

5. Talbot BH, Gifford JL, Peterson E, et al. The verbal overshadowing effect: influence on perception. Intuition 2008;4:12-18

6. Pellizon L, Brandimonte MA, Luccio R. The role of visual, spatial, and temporal cues in attenuating verbal overshadowing. Applied Cog Psych 2002;16:947-61

7. Meissner CA, Brigham JC, Kelley CM. The influence of retrieval processes in verbal overshadowing. Mem Cognit 2001;29:176-86

8. Huff M, Schwan S. Verbalizing events: overshadowing or facilitation? Mem Cognit 2008;36:392-402

9. Kebbell MR, Wagstaff GF. Hypnotic interviewing: the best way to interview eyewitnesses? Behavio Behav Sci Law 1998;16:115-29

10. Brown C, Lloyd-Jones TJ. Verbal overshadowing in a multiple face presentation paradigm: effects of description instruction. Applied Cog Psych 2002;16:873-85 